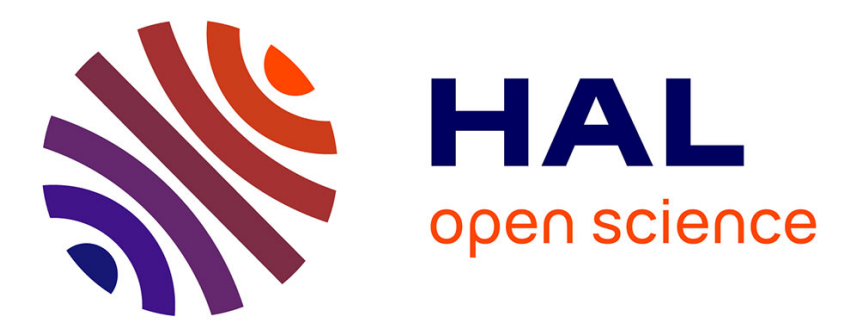

\title{
Identification of 2D Cracks by Elastic Boundary Measurements
}

\author{
Amel Ben Abda, Hend Ben Ameur, Mohamed Jaoua
}

\section{To cite this version:}

Amel Ben Abda, Hend Ben Ameur, Mohamed Jaoua. Identification of 2D Cracks by Elastic Boundary Measurements. [Research Report] RR-3438, INRIA. 1998. inria-00073252

\section{HAL Id: inria-00073252 \\ https://hal.inria.fr/inria-00073252}

Submitted on 24 May 2006

HAL is a multi-disciplinary open access archive for the deposit and dissemination of scientific research documents, whether they are published or not. The documents may come from teaching and research institutions in France or abroad, or from public or private research centers.
L'archive ouverte pluridisciplinaire HAL, est destinée au dépôt et à la diffusion de documents scientifiques de niveau recherche, publiés ou non, émanant des établissements d'enseignement et de recherche français ou étrangers, des laboratoires publics ou privés. 


\section{Identification of $2 D$ cracks by elastic boundary measurements}

Amel Ben Abda , Hend Ben Ameur and Mohamed Jaoua

$$
\mathbf{N}^{\circ} 3438
$$

June 5, 1998

THÈME 4 



\title{
Identification of $2 \mathrm{D}$ cracks by elastic boundary measurements
}

\author{
Amel Ben Abda * , Hend Ben Ameur ${ }^{\dagger}$ and Mohamed Jaoua $\ddagger$ \\ Thème 4 - Simulation et optimisation \\ de systèmes complexes \\ Projet ESTIME
}

Rapport de recherche $\mathrm{n}^{\circ} 3438$ - June 5, 1998 - 13 pages

\begin{abstract}
The purpose of this work is to identify 2D cracks by the mean of elastic boundary measurements. A uniqueness result is first proved in the general case, as well as the local lipschitzian stability in the case of line segment emergent cracks. In this last case, the search of the unique zero of the reciprocity gap functional related to the singular solution of the elasticity problem provides a fast algorithm to determine the unknown crack tip.
\end{abstract}

Key-words: Non destructive control, identification of cracks, identifiability, stability, reciprocity principle

(Résumé : tsvp)

* IPEST \& ENIT-LAMSIN, BP 37, 1002 Tunis-Belvédère, Tunisia

$\dagger$ FSB \& ENIT-LAMSIN

$\ddagger$ ENIT-LAMSIN \& UTC, Mathématiques Appliquées, BP 20529, 60205 Compiègne cedex, France - E-mail : Mohamed.Jaoua@utc.fr 


\section{Identification de fissures $2 \mathrm{D}$ par des mesures de frontière en élasticité}

Résumé : On se propose dans ce travail d'identifier des fissures $2 \mathrm{D}$ au moyen de mesures de déplacements effectuées sur une partie de la frontière. On montre d'abord un résultat d'unicité de la solution, sous réserve que le chargement imposé génère des facteurs d'intensité de contraintes non nuls. Puis, on démontre des résultats de stabilité locale lipschitzienee, seulement dans le cas de fissures droites débouchantes. Une méthode numérique rapide, basée sur la recherche du zéro de la fonction d'écart à la réciprocité, est ensuite proposée.

Mots-clé : Contrôle non destructif, identification de fissures, identifiabilité, stabilité, principe de réciprocité 


\section{Introduction}

We are interested in this paper in the identification of a surface breaking line segment crack by overspecified elastic data. Up to our knowledge, theoretical results on identifiability of cracks are seldom and are concerned mainly with the Laplace Equation ( i.e. steady state thermal equation or electrical conduction). The uniqueness (identifiability) result for a buried crack has been first given by Friedman and Vogelius [12]. They proved that two appropriate current fluxes, toghether with the corresponding voltages, are necessary and suffice to determine uniquely the crack. In the same paper, a partial stability result has been established. For the same problem, Alessandrini, Beretta and Vess 1 ela [1] proved a Lipshitz stability result.

Bryan and Vogelius [9], followed by Alessandrini and Diaz Valuenzia [3] examined the multiple crack problem, always in 2D situation. In [7], Andrieux, Ben Abda and Jaoua proved that the inverse surface breaking line segment crack problem is well-posed in Hadamard's sense (uniqueness and Lipschitz stability). More recently, Alessandrini and Di Benetto [2] studied the general 3D case for crack's determination by over determined electric data.

For the elastic case, when the crack is a priori known to be planar, and provided that complete data are available on the boundary $\partial \Omega$, Andrieux, Ben Abda and Bui [6] have proved a complete identification result based on the reciprocity gap principle introduced in [5]. The second section of this note concerns the uniqueness result, which is obtained, provided that the solution is not smooth at the crack tips. In the thermal case, such an assumption was only needed for stability purposes [7]. The third section is devoted to the stability result which uses, as for the thermal case, the domain derivative technique as a main tool. A restrictive assumption on the stress intensity factors is also needed. Finally, we present in the last section a fast algorithm to recover line segment cracks. The algorithm is based on the reciprocity gap functional, but used in a somewhat different way from [8] : the solution turns out to be a zero of the $R G$ functional applied to some singular solution of the elasticity system. A line search algorithm is then used to find out this zero, after the line has been determined by explicit formulae [6].

\section{Identifiability}

Let $\Omega$ be a smooth open domain of $\mathbb{R}^{2}$, with boundary $\partial \Omega$, and let $\Sigma$ be a crack in $\Omega$. The direct linear elastic problem, in the plane stress framework, is the following :

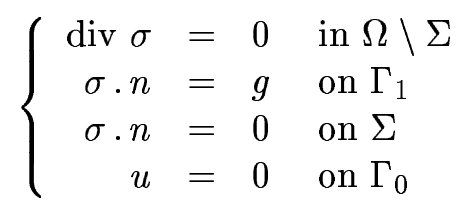

where $\Gamma_{1}$ and $\Gamma_{0}$ is a partition of $\partial \Omega$. The stress tensor $\sigma$ and the strain tensor $\varepsilon$ are related by the Hooke's constitutive law, the medium being supposed homogeneous and isotropic. Therefore :

$$
\varepsilon_{i j}(u)=\frac{1+\nu}{E} \sigma_{i j}-\frac{\nu}{E} \sigma_{l l} \delta_{i j}
$$

$E$ being the Young modulus, $\nu$ the Poisson ratio, and $\delta_{i j}$ the Kronecker symbol.

Let us also define the two following spaces :

$$
H:=\left\{\tau \in\left(L^{2}(\Omega)\right)^{4} ; \tau_{i j}=\tau_{j i} ; i, j=1,2\right\}
$$

and :

$\mathrm{RR} \mathrm{n}^{\circ} 3438$

$$
V_{0}:=\left\{v \in\left(H^{1}(\Omega)\right)^{2} ; v=0 \text { on } \Gamma_{0}\right\}
$$


Let $\Gamma_{a d}$ denote the set of admissible geometries and $M$ be some open subset of $\Gamma_{1}$ on which the measurements are realised. The identification process consists in putting some prescribed load $g$ on $\Gamma_{1}$, and measuring the displacement induced by it on $M$. The identifiability result is based on a unique continuation theorem for the elasticity system, similar to Holmgren's theorem for elliptic operators, and known as the Almansi Lemma [13]. Let us recall hereafter this result :

Lemma 1 (Almansi) Let $\Omega$ be a domain in $\mathbb{R}^{2}$ and $(\sigma, u)$ the solution of the linear elastic problem (1) (2) (with a source term eventually). Assume that there exists some open subset $L$ of $\partial \Omega$ such that :

$$
\left\{\begin{array}{rll}
\sigma \cdot n=0 & \text { on } L \\
u=0 & \text { on } L
\end{array}\right.
$$

Then, $\sigma \equiv 0$, and $u \equiv 0$ in $\Omega$.

The identifiability result is a consequence of this lemma, provided that the stress intensity factors, which are the coefficients of the two singular functions describing the behaviour of the solution at a crack tip, do not both vanish.

Theorem 1 (Identifiability) Let $\Sigma$ and $\tilde{\Sigma}$ be two cracks in $\Omega$, and let $u, \tilde{u}$ be the solutions of the direct problem (1) (2) on $\Omega_{\Sigma}$ and $\Omega_{\tilde{\Sigma}}$ for the loading $g$. Assume that the solution $u$ is not smooth at the vicinity of the crack tips (i.e. $u \notin H^{2}(\vartheta), \vartheta$ being any neighbourhood of the crack tips). Then, if $\Sigma$ and $\tilde{\Sigma}$ both lead to the same measured displacement on $M$, we have $\Sigma=\tilde{\Sigma}$.

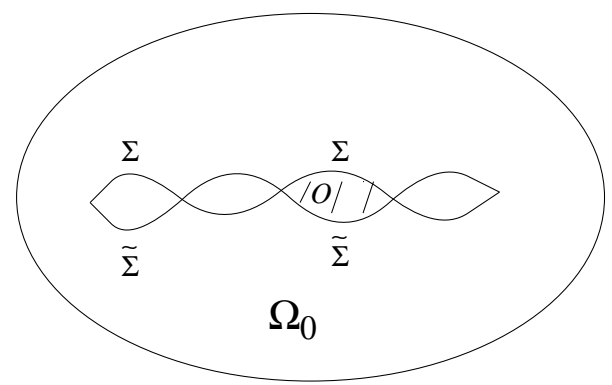

Figure 1: Two cracks with the same crack tips

Proof : Let $\Omega_{0}$ be the connected component of $\Omega \backslash(\Sigma \cup \tilde{\Sigma})$ containing $M . \tau:=\sigma-\tilde{\sigma}$ and $v:=u-\tilde{u}$ are therefore solution of the homogeneous elastic problem on $\Omega_{0}$ and $v$ vanishes on $M$. By the Almansi lemma, we derive that :

$$
\left\{\begin{array}{lll}
\sigma= & \tilde{\sigma} & \text { in } \Omega_{0} \\
u= & \text { in } \Omega_{0}
\end{array}\right.
$$

Since $u$ is not smooth at the vicinity of the crack tips of $\Sigma$, it follows that $\Sigma$ and $\tilde{\Sigma}$ have the same crack tips, otherwise $\tilde{u}$ would be singular at the vicinity of the crack tips of $\Sigma$, which are interior points of $\Omega \backslash \tilde{\Sigma}$.

Suppose now that $\Sigma \neq \tilde{\Sigma}$ and let $\mathcal{O}$ be a connected component of $\Omega \backslash \Omega_{0}$, which boundary is composed by a part of $\Sigma$ and a part of $\tilde{\Sigma}$. Then :

$$
\left\{\begin{aligned}
\operatorname{div} \sigma & =0 \text { in } \mathcal{O} \\
\sigma & =R(\varepsilon(u)) \text { in } \mathcal{O} \\
\sigma . n & =0 \text { on } \partial \mathcal{O} \cap \Sigma
\end{aligned}\right.
$$


Furthermore, $\sigma . n=\tilde{\sigma} . n=0$ on $\partial \Omega_{0} \cap \tilde{\Sigma}$. On the other hand, since $\sigma . n$ is continuous across $\tilde{\Sigma}$, we derive that $\sigma . n=0$ on $\partial \mathcal{O} \cap \tilde{\Sigma}$.

It follows that $u$ is a rigid displacement in $\mathcal{O}$. Let $U$ be this rigid displacement, defined by by $U(x)=a+b \times x$ in the whole $\Omega \backslash(\Sigma \cap \tilde{\Sigma})$. Hence, we get :

$$
\left\{\begin{aligned}
u-U & =0 \text { on } \tilde{\Sigma} \cap \partial \mathcal{O} \\
R \varepsilon(u-U) \cdot n & =0 \text { on } \tilde{\Sigma} \cap \partial \mathcal{O}
\end{aligned}\right.
$$

By the Almansi lemma, $u-U \equiv 0$ in $\Omega \backslash(\Sigma \cup \tilde{\Sigma})$, and therefore $\sigma \cdot n=0$ on $\partial \Omega_{0}$, which is in contradiction with the assumption that the load is not identically zero $(g \not \equiv 0)$.

Remark 1 : The same uniqueness result holds for $\mathcal{C}^{1}$ unknown boundaries, with fixed endpoints. In this case the only assumption needed is that the load $g \not \equiv 0$.

\section{$3 \quad$ Stability}

In this section, we shall focus on surface breaking line segment cracks. The main result needed is an expansion of the solution with respect to the geometry, and the tool used to obtain it is the domain derivative.

We define a family $F_{h}=I d+h \theta$ of diffeomorphisms mapping $\Omega \backslash \Sigma$ on $\Omega \backslash \Sigma_{h}$. Let now $\left(\sigma_{h}, u_{h}\right)$ be the solution of the elastic problem (1) (2) in $\Omega \backslash \Sigma_{h}$ and let $u^{h}=u_{h} \circ F_{h}$ and $\sigma^{h}=\sigma_{h} \circ F_{h}$. By [11], we have the following expansion :

Theorem $2\left(\sigma^{h}, u^{h}\right)$ has the following expansion in $H \times V_{0}$ :

$$
\left(\sigma^{h}, u^{h}\right)=\left(\sigma^{0}, u^{0}\right)+h\left(\sigma^{1}, u^{1}\right)+h o(h)
$$

where :

- $\left(\sigma^{0}, u^{0}\right)$ is the solution of the elastic problem in $\Omega \backslash \Sigma$

- $\lim _{h \rightarrow \infty}\|o(h)\|=0$ in $H \times V_{0}$

- $\left(\sigma^{1}, u^{1}\right)$ is the solution in $H \times V_{0}$ of the following problem :

$$
\left\{\begin{array}{l}
\forall \tau \in H \text { and } \forall v \in V_{0} \\
\int_{\Omega \backslash \Sigma} \frac{1+\nu}{E} \operatorname{Tr}\left(\sigma^{1} \tau\right)-\operatorname{Tr}(\sigma) \operatorname{Tr}(\tau)-\operatorname{Tr}\left(\tau \frac{\partial u^{1}}{\partial M}\right)=-\int_{\Omega \backslash \Sigma} \operatorname{Tr}\left(\tau \frac{\partial u^{0}}{\partial M} \frac{\partial v}{\partial M}\right) \\
-\int_{\Omega \backslash \Sigma} \operatorname{Tr}\left(\sigma^{1} \frac{\partial v}{\partial M}\right)=-\left[\int_{\Omega \backslash \Sigma} \operatorname{Tr}\left(\tau \frac{\partial v}{\partial M} \frac{\partial \theta}{\partial M}\right)-\int_{\Omega \backslash \Sigma} \operatorname{Tr}\left(\sigma^{1} \frac{\partial v}{\partial M}\right) d i v \theta\right]
\end{array}\right.
$$

Let $F$ be the interior endpoint of the crack, and let us choose a particular field $\theta$ :

$$
\theta(x, y)=\left\{\begin{array}{l}
\chi(x, y) \\
0
\end{array}\right.
$$

$\mathrm{RR} \mathrm{n}^{\circ} 3438$ 
where $\chi$ is a smooth fonction equal to 1 in a neighbourhood $\vartheta$ of $F$ which vanishes in the exterior of an other neighbourhood $\tilde{\vartheta}$ of $F . I d+h \theta$ describes then a variation of the length of the crack. To describe a variation of the angle, we shall use another field $\theta: \vartheta$ and $\mathcal{O}$ being two open neighbourhoods of $\Sigma$ in $\Omega$ such that: $\bar{\vartheta} \subseteq \mathcal{O} \subset \Omega \cup\{S\}$ and such that $\overline{\mathcal{O}} \cap \partial \Omega=\{S\}$ :

$$
\left\{\begin{array}{l}
\theta(x, y)=\left\{\begin{array}{l}
-y \text { in } \bar{\vartheta} \\
x
\end{array}\right. \\
\theta(x, y)=0 \text { in }(\Omega \backslash \Sigma) \backslash \overline{\mathcal{O}}
\end{array}\right.
$$
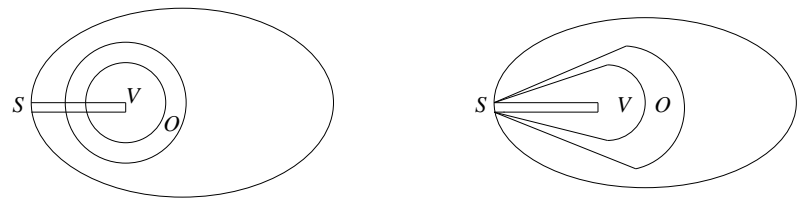

Figure 2: Neighbourhoods of the crack tip

The local stability result is then the following :

Theorem 3 Assume that $u$ is not smooth (i.e. $H^{2}$ ) near the crack tip. Then, for $\theta$ defined by (9), we have the stability result with respect to the length:

$$
\lim _{h \rightarrow 0} \frac{\left|u^{h}\right|_{M}-\left.u\right|_{M} \mid}{h}>0
$$

For $\theta$ defined by (10), and assuming that the stress intensity factor $K_{2}$ does not vanish, we get the stability result with respect to the angle variation:

$$
\lim _{h \rightarrow 0} \frac{\left|u^{h}\right|_{M}-\left.u\right|_{M} \mid}{h}>0
$$

Proof : Two kinds of stability have to be studied.

- Stability with respect to the length :

By the asymptotic expansion given by theorem 2, we have :

$$
\lim _{h \rightarrow 0} \frac{\left|u^{h}\right| M-\left.u\right|_{M} \mid}{h}=\left|u^{1}\right|_{L^{2}(M)}
$$

Suppose that $\left|u^{1}\right|_{L^{2}(M)}=0$. Then, because of the particular choice of $\theta$, the pair $\left(\sigma^{1}, u^{1}\right)$ satisfies :

$$
\left\{\begin{array}{rll}
\operatorname{div} \sigma^{1}= & & \text { in a neighbourhood of } \partial \Omega \\
\sigma^{1} n=0 & \text { on } M \\
u^{1}=0 & \text { on } M
\end{array}\right.
$$

where $\sigma^{1}=R\left(\varepsilon\left(u^{1}\right)\right)$. Therefore, the Almansi lemma gives that : $\left\{\begin{array}{l}u^{1} \equiv 0 \\ \sigma^{1} \equiv 0\end{array}\right.$ in a neighbouhood of $\partial \Omega$. Using the results in [11] we deduce that :

$$
-\frac{1}{2} \int_{\Gamma_{1}} g u^{1}=-\frac{1}{E}\left(K_{1}^{2}+K_{2}^{2}\right)=0
$$


and therefore

$$
K_{1}=K_{2}=0
$$

which contradicts the assumption we made on the stress intensity factors.

- $\underline{\text { Stability with respect to the angle : }}$

As for the first part of the proof, we derive from theorem 2

$$
\begin{gathered}
\lim _{h \rightarrow 0} \frac{\left|u^{h}\right|_{M}-\left.u\right|_{M} \mid}{h}=\left|u^{1}\right|_{L^{2}(M)} \\
\lim _{h \rightarrow 0} \frac{\left|\left(\left.\sigma^{h}\right|_{M}\right) n-\left(\left.\sigma\right|_{M}\right) \cdot n\right|}{h}=\left|\sigma^{1} \cdot n\right|_{L^{2}(M)}
\end{gathered}
$$

Suppose that $\left|u^{1}\right|_{L^{2}(M)}=0$ and consider a bole $B_{\delta}$, around the interior crack tip of $\Sigma$.

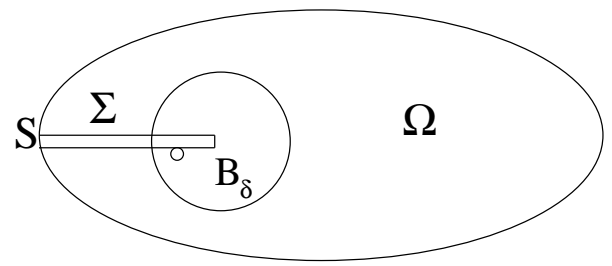

Figure 3: The neighbourhood $B_{\delta}$ of the crack tip

Denote by

$$
(\Omega \backslash \Sigma)_{\delta}=(\Omega \backslash \Sigma) \backslash B_{\delta}
$$

and let

$$
\left.\mathcal{S}:=\left\{v \in\left(H^{1}(\Omega)\right)^{2} ; \operatorname{div}\{\sigma(v))\right\}=0 \text { in } \Omega \backslash \Sigma\right\}
$$

where $\sigma(v)=R(\varepsilon(v))$.

The two following technical lemmas lead then to the contradiction.

Lemma 2 Let $v$ be a field in $\mathcal{S}$ and let $\tau=\sigma(v)$. Then $(\tau, v)$ satisfies :

$$
-\int_{\Omega} \operatorname{Tr}\left(\sigma^{0} \frac{\partial v}{\partial M} \frac{\partial \theta}{\partial M}\right)-\int_{\Omega} \operatorname{Tr}\left(\sigma^{0} \frac{\partial v}{\partial M}\right) d i v \theta+\int_{\Omega} \operatorname{Tr}\left(\tau \frac{\partial u^{0}}{\partial M} \frac{\partial \theta}{\partial M}\right)=\lim _{\delta \rightarrow 0} \int_{B_{\delta}} \operatorname{Tr}\left(\tau \frac{\partial u^{1}}{\partial M}\right)
$$

Lemma 3 Let $v$ be a field in $\mathcal{S}$. If

$$
\frac{\partial v^{1}}{\partial y}=\frac{\partial v^{2}}{\partial x}=0
$$

then

$$
\operatorname{grad}\left(\operatorname{Tr}\left(\sigma^{0} \frac{\partial v}{\partial M}\right)\right)-\operatorname{div}\left(\sigma^{0} \frac{\partial v}{\partial M}\right)=\operatorname{div}\left(\tau \frac{\partial u^{0}}{\partial M}\right)
$$

Let $v$ be defined on $\Omega$ by $v(x, y)=\left(\begin{array}{c}a x \\ b y\end{array}\right)$ where $a$ and $b$ are two real numbers satisfying :

$\mathrm{RR} \mathrm{n}^{\circ} 3438$

$$
\left(\frac{E}{1+\nu}+\frac{\nu}{1-\nu}\right) b+\frac{\nu}{1-\nu} a=0
$$


Such a field $v \in \mathcal{S}$. By lemma 3 , we get then :

$$
-\left(\int_{(\Omega \backslash \Sigma)} \operatorname{Tr}\left(\sigma^{0} \frac{\partial v}{\partial M} \frac{\partial \theta}{\partial M}\right)-\int_{(\Omega \backslash \Sigma)} \operatorname{Tr}\left(\sigma^{0} \frac{\partial v}{\partial M}\right) \operatorname{div} \theta\right)-\int_{(\Omega \backslash \Sigma)} \operatorname{Tr}\left(\tau \frac{\partial u^{0}}{\partial M} \frac{\partial \theta}{\partial M}\right)=\lim _{\delta \rightarrow 0} \int_{B_{\delta}} \operatorname{Tr}\left(\tau \frac{\partial u^{1}}{\partial M}\right)
$$

Due to our particular choice of $v, \tau$ is smooth, so that :

$$
\lim _{\delta \rightarrow 0} \int_{B_{\delta}} \operatorname{Tr}\left(\tau \frac{\partial u^{1}}{\partial M}\right)=0
$$

and

$$
\begin{aligned}
& \left(\int_{(\Omega \backslash \Sigma)_{\delta}} \operatorname{Tr}\left(\sigma^{0} \frac{\partial v}{\partial M} \frac{\partial \theta}{\partial M}\right)-\int_{(\Omega \backslash \Sigma)_{\delta}} \operatorname{Tr}\left(\sigma^{0} \frac{\partial v}{\partial M}\right) \operatorname{div} \theta\right)-\int_{(\Omega \backslash \Sigma)_{\delta}} \operatorname{Tr}\left(\tau \frac{\partial u^{0}}{\partial M} \frac{\partial \theta}{\partial M}\right) \\
& -\left(\int_{B_{\delta}} \operatorname{Tr}\left(\sigma^{0} \frac{\partial v}{\partial M} \frac{\partial \theta}{\partial M}\right)-\int_{B_{\delta}} \operatorname{Tr}\left(\sigma^{0} \frac{\partial v}{\partial M}\right) \operatorname{div} \theta\right)-\int_{B_{\delta}} \operatorname{Tr}\left(\tau \frac{\partial u^{0}}{\partial M} \frac{\partial \theta}{\partial M}\right)=0
\end{aligned}
$$

The functions to integrated on $B_{\delta}$ are in $L^{1}(\Omega \backslash \Sigma)$. Therefore,

$$
\lim _{\delta \rightarrow 0} \int_{B_{\delta}} \operatorname{Tr}\left(\sigma^{0} \frac{\partial v}{\partial M} \frac{\partial \theta}{\partial M}\right)-\int_{B_{\delta}} \operatorname{Tr}\left(\sigma^{0} \frac{\partial v}{\partial M}\right) \operatorname{div} \theta+\int_{B_{\delta}} \operatorname{Tr}\left(\tau \frac{\partial u^{0}}{\partial M} \frac{\partial \theta}{\partial M}\right)=0
$$

By Green's formula, we obtain :

$$
\lim _{\delta \rightarrow 0}\left[\begin{array}{c}
-\int_{(\Omega \backslash \Sigma)_{\delta}} \operatorname{Tr}\left(\tau \frac{\partial u^{0}}{\partial M}\right) \theta+\int_{\partial(\Omega \backslash \Sigma)_{\delta}} \bar{n} \tau \frac{\partial u^{0}}{\partial M} \theta-\int_{(\Omega \backslash \Sigma)_{\delta}} \operatorname{div}\left(\sigma^{0} \frac{\partial v}{\partial M}\right) \theta \\
+\int_{\partial(\Omega \backslash \Sigma)_{\delta}} \bar{n} \sigma^{0} \frac{\partial v}{\partial M} \theta-\int_{(\Omega \backslash \Sigma)_{\delta}} \operatorname{grad}\left(\operatorname{Tr}\left(\sigma^{0} \frac{\partial v}{\partial M}\right)\right) \theta-\int_{\partial(\Omega \backslash \Sigma)_{\delta}} \operatorname{Tr}\left(\sigma^{0} \frac{\partial v}{\partial M}\right) \bar{n} \theta
\end{array}\right]=0
$$

Since $\tau . n=0$ on $\Sigma, \sigma^{0} . n=0$ on $\Sigma$ and $\theta \equiv 0$ in a neighbourhood of $\partial \Omega$, we can conclude that the limits (when $\delta \rightarrow 0$ ) of $\int_{\partial(\Omega \backslash \Sigma)_{\delta}} \bar{n} \tau \frac{\partial u^{0}}{\partial M} \theta, \int_{\partial(\Omega \backslash \Sigma)_{\delta}} \bar{n} \sigma^{0} \frac{\partial v}{\partial M} \theta, \int_{\partial B_{\delta}} \bar{n} \tau \frac{\partial u^{0}}{\partial M} \theta$ and $\int_{\partial B_{\delta}} \bar{n} \sigma^{0} \frac{\partial v}{\partial M} \theta$ are all equal to zero.

Therefore, (29) is equivalent to :

$$
\lim _{\delta \rightarrow 0} \int_{\partial(\Omega \backslash \Sigma)_{\delta}} \operatorname{Tr}\left(\sigma^{0} \frac{\partial v}{\partial M}\right) \bar{n} \theta=0
$$

Using the previous arguments, we deduce that :

$$
\int_{\Sigma^{+} \cup \Sigma^{-}} \operatorname{Tr}\left(\sigma^{0} \frac{\partial v}{\partial M}\right) \bar{n} \theta=0
$$

and hence

$$
\int_{\Sigma}\left(a\left[\sigma_{11}\right]+b\left[\sigma_{22}\right]\right)(l+r)=0
$$

where [ ] denotes the jump of the function between the brackets across the crack $\Sigma$. Since $\left[\sigma_{11}\right]=$ $\frac{-4 K_{2}}{(2 \pi r)^{\frac{1}{2}}}$ and $\left[\sigma_{22}\right]=0$, we obtain that

$$
\frac{-4 K_{2}}{(2 \pi)^{\frac{1}{2}}} \int_{0}^{l} \frac{(l+r)}{r^{\frac{1}{2}}} d r=0
$$

and therefore $K_{2}=0$, which is again in contradiction with our assumption. 


\section{$4 \quad$ Numerical issues and comments}

To solve numerically the problem under consideration, let us assume that the overspecified data is given on the whole external boundary of $\Omega$. We use the reciprocity gap principle introduced in [5] in the framework of the thermal conductivity and generalised in [6] to the elastic case.

The reciprocity gap functional for the considered problem is then defined on the space $\mathcal{S}$ by:

$$
R G(v)=\int_{\partial \Omega} g v-u\{R(\varepsilon(v)) \cdot n\}
$$

which, by Green's formula, is also :

$$
R G(v)=\int_{\Sigma}[u]\{R(\varepsilon(v) \cdot N\}
$$

$[u]$ being the jump of $u$ accross $\Sigma$.

This last expression of $R G$ gives rise to the explicit reconstruction of the line supporting the crack, by calculating its normal vector $N[6]$. The reciprocity gap principle can be exploited beyond to reconstruct $\Sigma$ ( that is to find its length). For a given crack $\tilde{\Sigma}$ of length $\tilde{\lambda}$, the idea is to select a particular singular displacement field corresponding to a divergence free stress tensor (in $\Omega$ ), and such that the normal component of the related stress tensor vanishes on $\tilde{\Sigma}$. The singular function $\tilde{S}$, defined in polar coordinates (with respect to the crack tip of $\tilde{\Sigma}$ ) by :

$$
\tilde{S}(\rho, \theta)=\rho^{\frac{1}{2}} \psi(\theta)
$$

where

$$
\psi(\theta)=\left\{\begin{array}{l}
\frac{1}{(2 \Pi)^{\frac{1}{2}} E} \cos \left(\frac{\theta}{2}\right)(3-\nu(1+\nu) \cos (\theta)) \\
\frac{1}{(2 \Pi)^{\frac{1}{2}} E} \sin \left(\frac{\theta}{2}\right)(3-\nu(1+\nu) \cos (\theta))
\end{array}\right.
$$

is divergence free and satisfies $\sigma(\tilde{S}) . N=0$ on both sides of the crack $\tilde{\Sigma}$. By calculating the reciprocity gap functional (34) for this field, we define a function of the length $\tilde{\lambda}$ :

$$
r g(\tilde{\lambda})=R G(\tilde{S})
$$

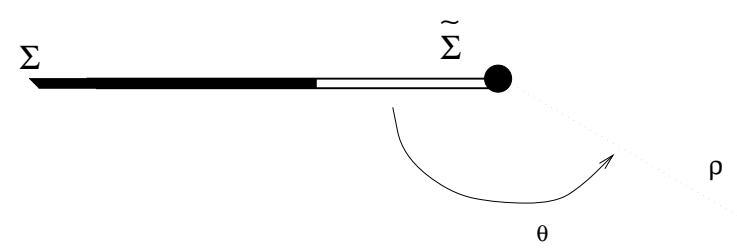

Figure 4: The polar coordinates with respect to the crack $\tilde{\Sigma}$

$\Sigma$ being the crack to identify, and $\lambda$ its length, by the Maxwell-Betti reciprocity principle, it is clear that, $r g(\tilde{\lambda})=0$ if $\tilde{\Sigma}=\Sigma$. The location of the second endpoint of $\Sigma$ can then be found by seeking a zero of $r g$. 
Let us check the behaviour of this function at the vicinity of its zero, for example for $\tilde{\lambda}>\lambda$ (the case $\tilde{\lambda}<\lambda$ works exactly the same way, by exchanging the roles of $u$ and $\tilde{S}$, and those of $\Sigma$ and $\tilde{\Sigma})$. Then, we have :

$$
r g(\tilde{\lambda})=\int_{\tilde{\Sigma} \backslash \Sigma}[\tilde{S}]\{R(\varepsilon(u)) \cdot N\}
$$

Since the crack intensity factors do not both vanish, $R(\varepsilon(u)) . N(\mu)$ can be expanded as follows on $\tilde{\Sigma} \backslash \Sigma$ $(\mu \in[\lambda, \tilde{\lambda}]):$

$$
R(\varepsilon(\tilde{S})) . N(\mu)=\left(\frac{1}{(2 \Pi)^{\frac{1}{2}} E}\left(\frac{2 \nu}{1+\nu}+\frac{E}{1+\nu}\right)\right)(\mu-\lambda)^{-\frac{1}{2}}+c(\mu-\lambda)^{\frac{1}{2}}+\ldots
$$

Hence, by an easy computation,we get the following local expansion of the $r g$, at the vicinity of $\lambda$ :

$$
r g(\tilde{\lambda})=\beta K_{1}(\tilde{\lambda}-\lambda)+(\tilde{\lambda}-\lambda) O(\tilde{\lambda}-\lambda)
$$

where $\beta \neq 0$ is a non vanishing constant.

(39) shows that a non vanishing opening mode stress intensity factor insures a non vanishing derivative of $r g$ with respect to the crack length in the neighbourhood of its solution. This is due to our choice of the opening mode singular function to define $\mathrm{rg}$. Choosing the shear mode singular function would lead to an expansion involving $K_{2}$ instead of $K_{1}$. Anyway, nonvanishing stress intensity factors have important effects on the local behaviour of the $r g$ function, and therefore on the performance of the line search algorithm used to compute its zero.

The numerical trials have been performed using synthetic data calculated by a direct finite element method using the Modulef library.

Figure 5 shows how the $r g$ function looks like in various situations, depending on the crack lengths, for a prescribed load. Any line search algorithm, such as the fixed point one, may be used to find out the zero of $r g$. Only few iterations are necessary to converge to a satisfactory solution.

Figure 6 shows how, by choosing an appropriate load, one can improve the identification process (Bryan and Vogelius [10]).

An important issue to be discussed is related to the behaviour of the algorithm with respect to noise on the measured data. Actually, it turns out to be amazingly robust : Up to $25 \%$ noise, the zero is only slightly perturbated. At a $40 \%$ noise level, the $\mathrm{rg}$ function has more than a zero, but they remain not far from the actual one. Things become bad at $60 \%$ and $80 \%$ noise level (see figure), which is somewhat expected.

Figure 8 displays the error on the crack tip location with respect to the crack length, and with respect to the noise level on the data.

Issues remaining to be discussed concern the interior crack identification. It seems possible to generalize the algorithm to such situations, but this have not been done yet. A full numerical analysis of the algorithm, including an error analysis and a characterization of the solution of the identification problem as the unique zero of the $r g$ function, is also an interesting problem to be studied. 

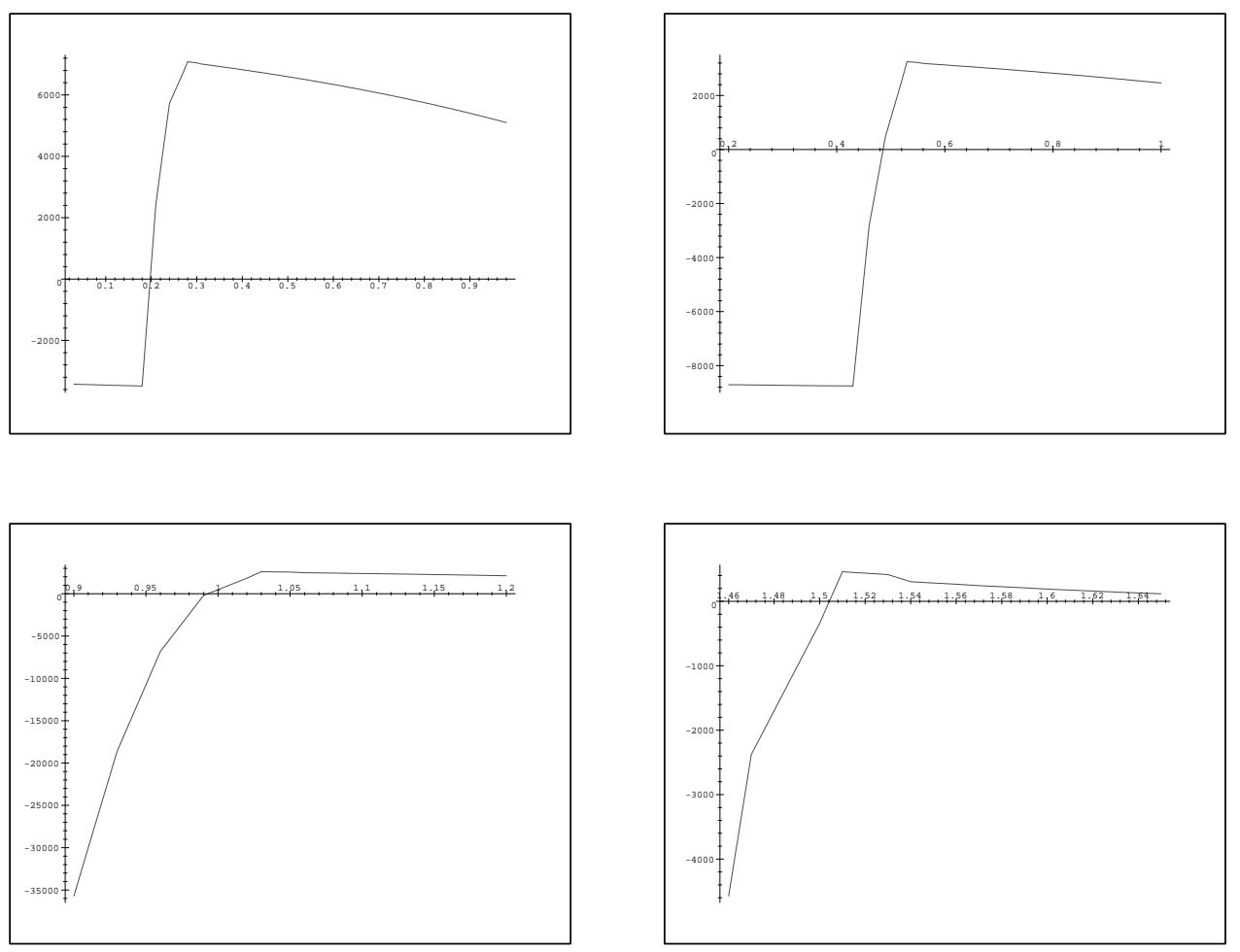

Figure 5: Zeros of the function rg for various crack lengths $\lambda ; \lambda=0.2 ; \lambda=0.5 ; \mathrm{SW}: \lambda=1.0, \theta=; \mathrm{SE}: \lambda=1.5$
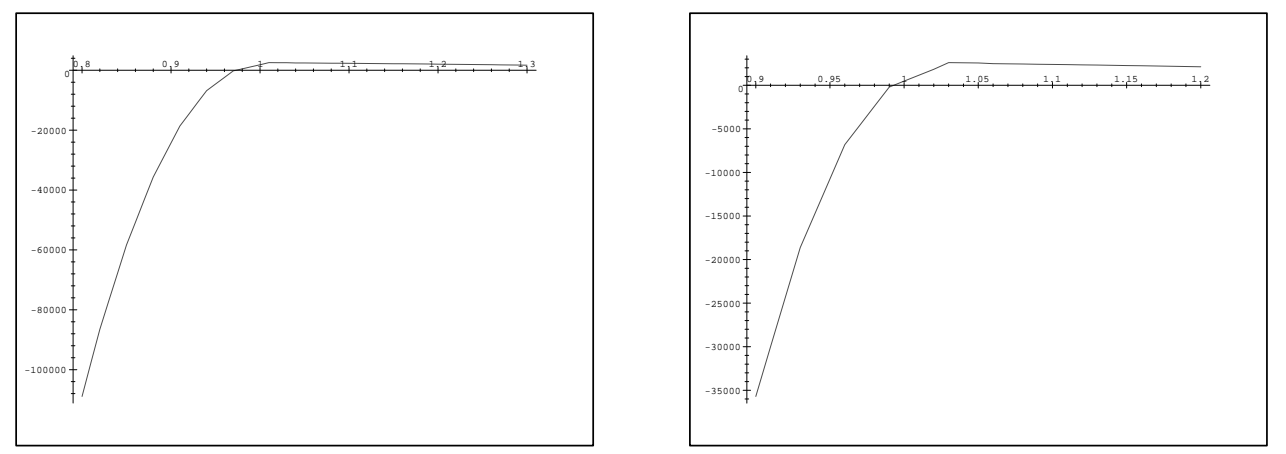

Figure 6: Improving the identification by choosing an appropriate loading (actual length $=1$.) 

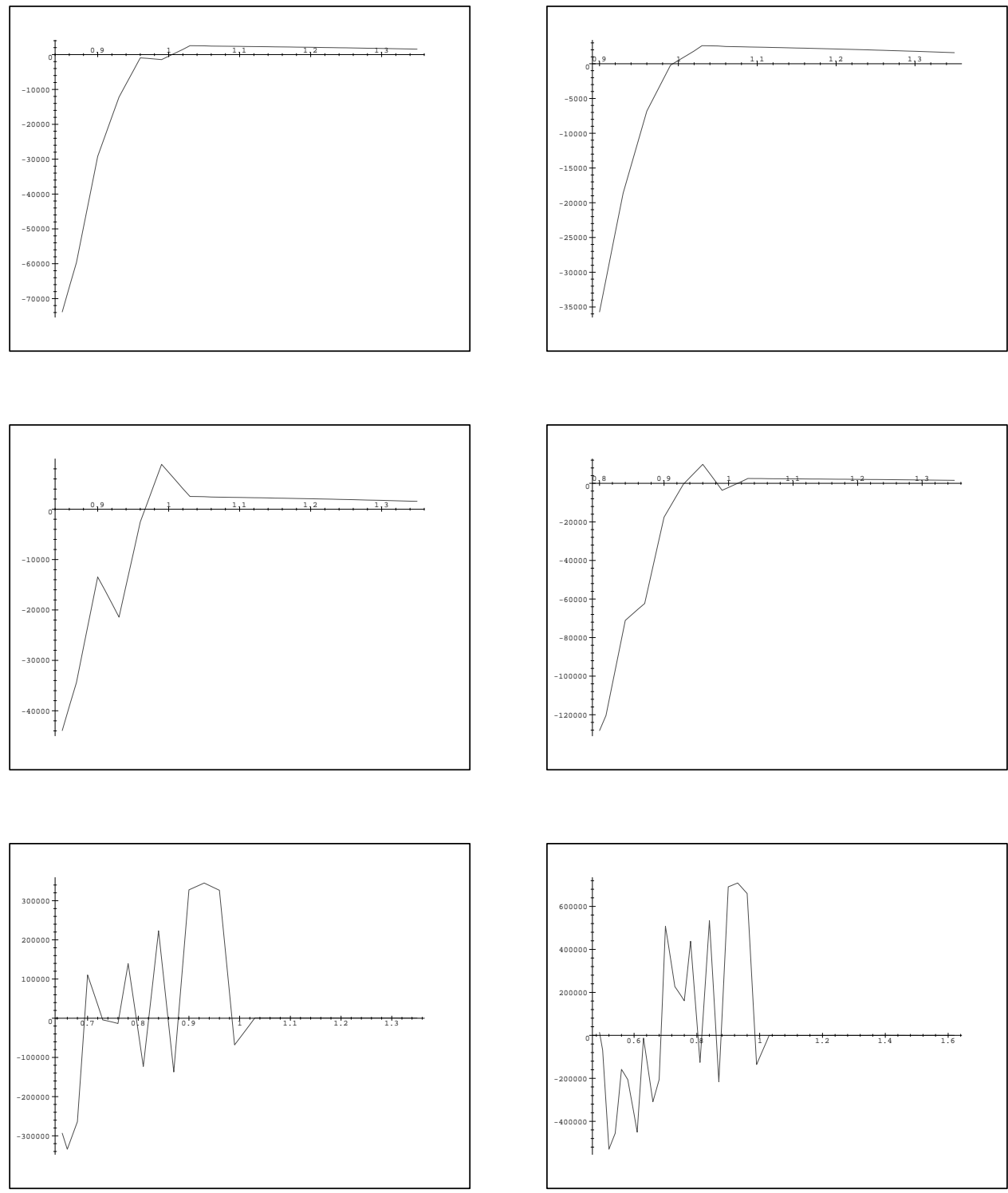

Figure 7: The rg function for noisy data : $5 \%, 10 \%, 20 \%, 40 \%, 60 \%$ and $80 \%$
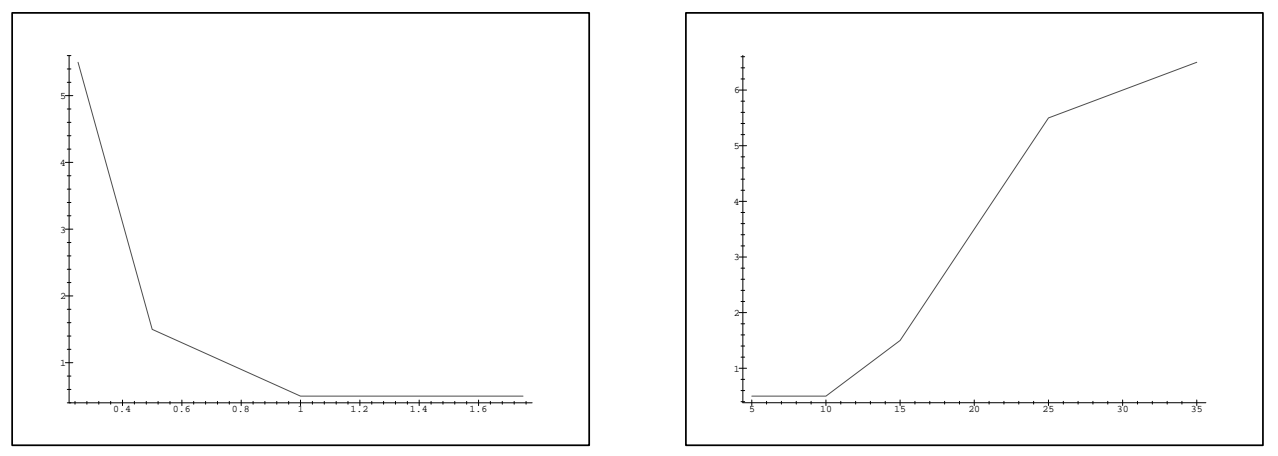

Figure 8: Error with respect to the crack length, and to the noise level 


\section{References}

[1] G. Alessandrini, E. Beretta, S. Vessella (1996) : Determining linear cracks by boundary measurements-Lipschitz stability., SIAM J. Math. Anal., 27, pp. 361-375

[2] G. Alessandrini, E. Di Benedetto (1997) : Determining 2-Dimensional cracks in 3-Dimensional Bodies: uniqueness and stability., Indiana Univ. Math. J., 46, \# 1, pp. 1-82

[3] G. Alessandrini, A. Diaz Valenzuela (1996) : Unique determination of multiple cracks by two measurements., SIAM J. Contr. Opt., 34, pp. 913-921

[4] S. Andrieux (1995) : Fonctionnelles d'écart à la réciprocité généralisée et identification de fissures par des mesures surabondantyes de surface, C. R. Acad. Sci. Paris, 320, Série I, pp. 1553-1559

[5] S. Andrieux, A. Ben Abda (1996) : Identification of planar cracks by complete overdetermined data : inversion formulae, Inverse problems, 12, pp. 553-563

[6] S. Andrieux, A. Ben Abda, H.D. Bui (1997) : Sur l'identification de fissures planes via le concept d'écart à la réciprocité en élasticité., C. R. Acad. Sci. Paris, 46, Série I, pp. 1431-1438

[7] S. Andrieux, A. Ben Abda, M. Jaoua (1998) : On the inverse emergent plane crack problem., Math. Meth. in the Appl. Sc., to appear

[8] T. Bannour, A. Ben Abda, M. Jaoua (1997) : A semi-explicit algorithm for the reconstruction of $3 D$ planar cracks, Inverse Problems, 13, pp. 899-917

[9] K. Bryan, M. Vogelius (1992) : A uniqueness result concerning the identification of a collection of cracks from finitely many electrostatic boundary measurements ., SIAM J. Math. Anal., 23, \# 4, pp. $950-958$

[10] K. Bryan, M. Vogelius (1994) : A computational algorithm to determine crack locations from electrostatic boundary measurements, Int. J. Eng. Sci., 32

[11] P. Destuynder, M. Jaoua. (1981) : Sur une interprétation de l'integrale de Rice en théorie de la rupture fragile, Math. Meth. in the Appl. Sc., 3, \# 4, pp. 70-87

[12] A. Friedman, M. Vogelius (1989) : Determining cracks by boundary measurements, Indiana Univ. Math. J., 38

[13] N.I. Mushkelishvili (1963) : Some basic problems of mathematical theory of elasticity, Noordhoff 
Unit'e de recherche INRIA Lorraine, Technopôle de Nancy-Brabois, Campus scientifique, 615 rue du Jardin Botanique, BP 101, 54600 VILLERS LÈS NANCY

Unit'e de recherche INRIA Rennes, Irisa, Campus universitaire de Beaulieu, 35042 RENNES Cedex

Unit'e de recherche INRIA Rhône-Alpes, 655, avenue de l'Europe, 38330 MONTBONNOT ST MARTIN

Unit'e de recherche INRIA Rocquencourt, Domaine de Voluceau, Rocquencourt, BP 105, 78153 LE CHESNAY Cedex

Unit'e de recherche INRIA Sophia-Antipolis, 2004 route des Lucioles, BP 93, 06902 SOPHIA-ANTIPOLIS Cedex

\section{Éditeur}

INRIA, Domaine de Voluceau, Rocquencourt, BP 105, 78153 LE CHESNAY Cedex (France)

http://www.inria.fr

ISSN 0249-6399 